\title{
Research Progress of Anti-tumor Effects of Curcuma zedoaria and its Active Ingredients Through Immune Regulation Mechanism
}

\author{
Zhuli Hou', Gang Fang2* \\ ${ }^{1}$ Department of Medicine, Hubei University for Nationalities, Enshi 445000, Hubei Province, China \\ ${ }^{2}$ Guangxi Key Laboratory of Applied Fundamental Research of Zhuang Medicine, Guangxi Traditional Chinese Medical \\ University, Nanning 530200, Guangxi Province, China
}

*Corresponding author: Gang Fang, fglzyznn@yeah.net

\begin{abstract}
The continuous increase in the incidence rate of various fatal malignant tumors in the recent years warrants an imperative search for medications or drugs with obvious anti-tumor effects and reliable curative effects. Previous studies have found that Curcuma zedoaria and its active ingredients, such as turmeric oil, curcumol, and $\beta$-elemene, have obvious antitumor effects, and they do not have the adverse reactions and side effects seen in the anti-tumor drugs of Western medicine. Based on the review and inductive analysis of related literature, we summarize in the present article the results of some researchers who investigated the anti-tumor effects of Curcuma zedoaria and its active ingredients through the immune regulation mechanism.
\end{abstract}

Keywords: Malignant tumor; Curcuma zedoaria and its active ingredients; Immune regulation; Mechanism; Anti-tumor effect

Publication date: July 2021; Online publication: July 31, 2021

\section{Introduction}

Curcuma zedoaria is a plant that belongs to the ginger Zingiberaceae. It is warm in nature and bitter in taste, and have special functions or effects on liver and spleen. This herb has the function of activating blood to resolve stasis, and eliminating stagnated food and relieving pain. Based on the Treatise on the Nature of Medicinal Herbs (transliterated as Yao Xing Lun in Chinese), "Curcuma zedoaria can improve blood and $q i$, and cure the heart pain of a lady, combat the hypochondriac or abdominal mass and cold wind. Take the ground medicine with alcohol and vinegar to attain effective therapeutic outcome." Curcuma zedoaria is one of the anti-cancer Chinese herbal medicines discovered by medical workers in the early period during the Cultural Revolution. Its application in the clinical setting has achieved very good results. The anti-tumor effect of Curcuma zedoaria and its active ingredients is mediated by enhanced immune function and regulated cytokine levels, as well as the development of Curcuma zedoaria-based tumor vaccines to enhance the immunogenicity of tumor cells. This article reviews the immune function, cytokines, active immunity through tumor vaccine, etc. to explore the principle and mechanism of Curcuma zedoaria and its active ingredients in exerting anti-tumor effects through immune regulation.

\section{Enhanced immune function}

Lymphocytes are a type of white blood cells. They are produced by lymphoid organs. They are currently the smallest known white blood cells, belonging to the cell system with immune recognition function. 
According to their migration trend, surface protein molecules and different functions, lymphocytes can be divided into antigen-specific T lymphocytes (also known as T cells), antigen-specific B lymphocytes (also known as B cells), and natural killer cells (NK cells).

Macrophages are white blood cells located in tissues. They not only participate in non-specific defenses (innate immunity), but also specific defense (cellular immunity) in vertebrates. The main immune function and role of macrophages is to phagocytize the debris produced as a result of cell death or lysis, and engulf pathogens that invade the body in the form of fixed cells or free cells (that is, phagocytosis and digestion). Through these processes, related lymphocyte cells and other related immune mechanisms are activated, so that they can engender an immune response against existing pathogens and other substances or those that invade on next occasion. Moreover, macrophages also release various inflammatory substances, such as tumor necrosis factor (TNF), to activate the gene of nuclear factor kappa B (NF-кB) so as to enhance immune regulation.

Chen et al. found that Curcuma zedoaria polysaccharide (CZP) has a strong effect of enhancing the proliferation of lymphocytes and the phagocytic activity of macrophages ${ }^{[1]}$. At the same time, they also confirmed through in vivo experimental studies that CZP could increase the spleen index of tumor-bearing mice, thus proving that the application of Curcuma zedoaria and its active ingredient (i.e. CZP) can result in the increase of the proliferative ability of lymphocytes, the phagocytic activity of macrophages, as well as the spleen index of tumor-bearing mice, which can be construed as indicators of enhanced immune function.

Yao established a mouse model of $\mathrm{H} 22$ liver cancer which were administered with $\beta$-elemene through intraperitoneal injection, and found that the H22 liver cancer model mice had increased T lymphocyte transformation rate, and $\beta$-elemene also promoted the production of $\mathrm{T}$ lymphocytes in $\mathrm{H} 22$ liver cancer model mice ${ }^{[2,3]}$. According to the experimental studies conducted by Lin et al., a variety of immune-related co-stimulatory factors on the surface of macrophages can be enhanced by $\beta$-elemene, resulting in enhanced presentation of foreign antigen by macrophages ${ }^{[3,4]}$. This could indirectly activate $T$ cells to participate in the immune response, while $\beta$-elemene can also enhance the phagocytic ability of macrophages. Tian et al. treated patients with advanced non-small cell lung cancer with elemene emulsion added to chemotherapy and found that the concentration of $\mathrm{CD}^{+}$and $\mathrm{CD}^{+}$cells in the patients was increased after treatment, and the ratio of $\mathrm{CD}^{+} / \mathrm{CD}^{+}$cells became significantly higher ${ }^{[3,5]}$. This proves that Curcuma zedoaria and its active ingredient (i.e. $\beta$-elemene) can increase the transformation rate of $\mathrm{T}$ lymphocytes and promote the generation of $\mathrm{T}$ lymphocytes. It can facilitate in the enhanced expression of co-stimulatory factors on the surface of immune-related macrophages, thereby enhancing the presentation of foreign antigens by macrophages, improving the phagocytic activity of macrophages, and also activating the immune regulation mechanism involving related $\mathrm{T}$ cells. The increased concentration of $\mathrm{CD}^{+}$and $\mathrm{CD} 4^{+}$cells and the ratio of $\mathrm{CD}^{+} / \mathrm{CD}^{+}$cells represent the overall immune function of the body. Curcuma zedoaria and its active ingredient, such as $\beta$-elemene, can significantly increase the concentration of $\mathrm{CD}^{+}$and $\mathrm{CD}^{+}$cells and $\mathrm{CD} 4^{+} / \mathrm{CD}^{+}$cell ratio. Judging from this line of evidence, Curcuma zedoaria and its active ingredient, $\beta$ elemene, can be used to treat tumors using the above-mentioned methods.

Dendritic cells in the body belong to a type of cells that are specially used for antigen presentation (antigen presenting cells, APC). They work by efficiently ingesting, processing, and presenting antigens. Dendritic cells are divided into two types: immature dendritic cells and mature dendritic cells. The more prominent feature of immature dendritic cells is that they have relatively strong migration ability compared to mature dendritic cells, whereas the more prominent feature of mature dendritic cells is that they can effectively activate the primitive T cells that exist in the body; therefore, mature dendritic cells play a central role in the immune response system ${ }^{[6]}$.

Toll-like receptors (TLRs) is a molecule that has become known by people since the 21 st century, and 
belongs to a type of pattern recognition receptor for pathogen-associated molecular patterns (PAMPs), which are closely related to pathogens in innate immunity ${ }^{[7-10]}$. The main role of TLRs is to participate in some essential processes related to innate immune function. They also play a very crucial role when foreign pathogenic microorganisms invade the body's immune system ${ }^{[7]}$. Initially, when TLRs were first discovered, this type of receptor was simply considered for being unique only to the immune system, but Huang et al. found that TLRs are also expressed in tumor cells, not just unique to the immune system ${ }^{[7,11]}$. Based on previous studies, Sato et al. also conducted a detailed summary, recording the expression of TLRs in different human tumor tissues, and found that different human tumor tissues have differential expression of TLRs; this attribute is not only unique to the immune system ${ }^{[7,12]}$.

Idoyaga et al. ${ }^{[6,7,13,14]}$ found that the maturation of dendritic cells can be prevented by tumor cells by regulating TLR and its ligands, while the main role of mature dendritic cells is to activate the immune system and play a role by effectively activating the primitive $\mathrm{T}$ cells that exist in the body, playing an important role in the immune response system ${ }^{[6]}$. Undoubtedly, this finding provides a new mechanism for tumor immune escape. The experimental studies of Wang et al. also confirmed that the macrophage inflammatory protein MIP-3A (also known as CCL20) can recruit a large number of immature dendritic cells into tumor tissues, and TLR4 signal promotes the secretion of a large amount of MIP-3A from the tumor cells, thereby creating an opportunity for tumor immune escape ${ }^{[7,15,16]}$. Xu et al. showed that Curcuma zedoaria oil can also inhibit the expression of TLR2 and TLR4 proteins in rectal cancer cells ${ }^{[17]}$, which is consistent with previous reports ${ }^{[18]}$, suggesting that Curcuma zedoaria and its active ingredient, i.e. Curcuma zedoaria oil, also have an immune-enhancing effect. The main components of Curcuma zedoaria include Curcuma zedoaria oil and curcumin, while there are up to dozens of main components in Curcuma zedoaria oil, including curcumol, curdione, $\beta$-elemene, curcumenol, isocurcumenol, dehydrocurdione, turmeric diol, curcumenone, bisacurone, and curcumene. This proves that Curcuma zedoaria and its active ingredient, i.e. Curcuma zedoaria oil, can affect dendritic cells by affecting the expression of TLR and its ligands, and also affect the overall immune function of the body by influencing dendritic cells.

Another type of innate immune cells in the body is the NK cells, whose role in the immune mechanism ${ }^{[3]}$ is to recognize susceptible tumor cells through recognition of the receptors on cell surface. NK cells are tightly combined with the special target structure of the cell surface receptor, and then releases perforin or cytolysin (cytolysin) into the tumor cell to perforate the cell membrane, causing the leakage of cytoplasm out of the tumor cell and resulting in the death of susceptible tumor cells. Moreover, NK cells can also release various cytokines involved in immune response, such as interferon gamma (IFN- $\gamma$ ), interleukin (IL)1, and IL-2, which greatly strengthen and expand the role of NK cell in immune surveillance ${ }^{[3,19]}$. Previous studies ${ }^{[3,20]}$ have shown that elemene can significantly restore the immune function of tumor-bearing mice, which have low immune function caused by chemotherapy, and it can significantly promote and increase the activity of NK cells and the immune cells. The ability to produce IL-2 indicates that Curcuma zedoaria and its active ingredient, i.e. elemene, can contribute to the body's immune mechanism by increasing the activity of NK cells and enhancing the production of IL-2.

\section{Impact on the level of cytokines}

IL-2 is one of the most important cytokines in the body, and plays a very key role as an indispensable member of the cytokine network. It mainly exerts biological functions by promoting the division of lymphocytes in the body, assisting in enhancing the killing effect of immune cells on tumor cells, and assisting in the production of a series of antibodies ${ }^{[21]}$.

TNF- $\alpha$ is a kind of monocyte factor that functions to elicit apoptosis and necrosis of tumor cells, and also activates lymphokine in the immune system ${ }^{[6]}$. At the same time, TNF- $\alpha$ can also interact with other 
cytokines, such as IL-2, to attain a series of immune effects ${ }^{[22]}$.

IFN- $\gamma$ is a cytokine that regulates the immune system in the body. It has a role in multiple necessary processes of the immune system in the body, mainly by activating macrophages and upregulating the expression of main histocompatibility complex (MHC) in the body ${ }^{[23]}$, through which the body's immune function is influenced.

Through an experimental study, Chen et al. found that CZP can significantly increase the concentration levels of IL-2, TNF- $\alpha$ and IFN- $\gamma$, thereby exerting an inhibitory effect on Lewis lung cancer ${ }^{[1]}$. The experimental study of Chen et al. found that Curcuma zedoaria oil can significantly upregulate the concentration levels of IL-2 and IFN- $\gamma$, thereby diminishing the immune exhaustion caused by tumors ${ }^{\text {[24] }}$. The above-mentioned studies have proven that Curcuma zedoaria and its active ingredient, such as CZP, can increase the concentration of IL-2, TNF- $\alpha$ and IFN- $\gamma$ in the body, thereby enhancing the body's immune function via the regulation of the level of cytokines.

Transforming growth factor-beta 1 (TGF- $\beta 1$ ) can extensively inhibit the patient's own immune function in a variety of mechanisms of tumor immune escape, and more importantly, tumors and other cell lines can secrete TGF- $\beta 1^{[25]}$. In an experiment, Xu et al. found the Curcuma zedoaria oil after 24 hours in SW1463 cells decreased the expression of TGF- $\beta 1$ in the cells gradually with the increase of the drug concentration of Curcuma zedoaria oil, suggesting that Curcuma zedoaria and its active ingredient, i.e. Curcuma zedoaria oil, can exert immune effects by inhibiting the expression of TGF- $\beta 1$ in cells ${ }^{\text {[26] }}$.

IL-10 was the first negative regulator that has been reported to have an inhibitory effect in the body. It can inhibit the function and activity of T cells and macrophages ${ }^{[27]}$. C-Raf (Raf-1) belongs to a class of immune cytokine known as the mitogen-activated protein kinase (MAPK) factor. When C-Raf (Raf-1) is activated, it is often followed by the activation of the related MAPK signaling pathway. The activation of this pathway will result in the disruptions of the cell cycle, which in turn lead to the formation of a tumor [28]. Through experiment study, Xu et al. found that Curcuma zedoaria and its active ingredient, such as Curcuma zedoaria oil, can significantly reduce the levels of IL-10 and C-Raf (Raf-1) in rectal cancer cells, suggesting that Curcuma zedoaria and its active ingredient, such as Curcuma zedoaria oil, play immunomodulatory roles through the suppression of the expression of IL-10 and C-Raf (Raf-1) in cells ${ }^{[17]}$.

\section{Active immunity through tumor vaccine}

The fundamental research of specific active immunotherapy for tumors has been going on for almost a century. Specific active immunotherapy for tumors is a method that uses tumor cells (tumor antigens) or modified tumor cell materials to induce the body to produce specific antibodies that are used prevent the growth, metastasis and recurrence of tumors. In recent years, cancer researchers have begun to try to extract monomers from traditional Chinese medicine or modify and construct tumor cells with certain nonpathogenic viruses to improve the body's immunogenicity and tumor-killing effect, thus providing promising approaches of using specific active immunotherapy for tumors ${ }^{[29]}$.

$\mathrm{Xu}$ et al. found that the use of curcumol mixed with tumor cells as a vaccine can enhance the immunogenicity of tumor cells and stimulate the body to produce related specific cellular immunity, thereby exerting a powerful tumor-killing effect ${ }^{[30]}$. This provides a new idea of active immunity through tumor vaccine based on Curcuma zedoaria and its active ingredients.

Dendritic cell is the most powerful antigen-presenting cell known so far. It can extract and process living cells with soluble antigens. The antigens are presented to T lymphocytes through dendritic cells, and participate in the differentiation of $\mathrm{T}$ cell subsets and innate immune response. Therefore, tumor vaccines based on dendritic cells have become a research hotspot in biological therapy for tumor ${ }^{[31]}$.

Kang et al. found that the tumor vaccine made by using $\beta$-elemene, the active ingredient of Curcuma zedoaria, combined with dendritic cells can significantly promote the proliferation of mouse spleen cells 
and the secretion of IFN- $\gamma^{[32]}$. It is important to note that the index of spleen cells to a large extent represents the strength of non-specific immune function, and IFN- $\gamma$ is a cytokine that regulates the immune system in the body mainly by activating macrophages and upregulates the expression of MHC molecules in the body

${ }^{[23]}$, thereby regulating the immune function of the body. It was also found that the tumor vaccine made of $\beta$-elemene combined with dendritic cells can significantly inhibit the growth of subcutaneous xenograft tumors in tumor-bearing mice with liver cancer, and obviously prolong the survival time of tumor-bearing mice; after analysis, it is believed that the antigen presentation function of dendritic cells can be enhanced by Curcuma zedoaria and its active ingredient, i.e. $\beta$-elemene ${ }^{[3]}$. This indicates that $\beta$-elemene, the active ingredient of Curcuma zedoaria, can be use in combination with dendritic cells to make tumor vaccines to promote the proliferation of mouse spleen cells and the secretion of IFN- $\gamma$ so as to regulate the function of the immune system and exert anti-tumor effect.

\section{Conclusion}

In summary, Curcuma zedoaria and its active ingredients can exert anti-tumor effects by enhancing the immune function and regulating the concentration of cytokines, as well as through the development of Curcuma zedoaria-based tumor vaccines to enhance the immunogenicity of tumor cells. The anti-tumor property of this Chinese herbal medicine has been proven effective, and possess great clinical application value and development prospects. However, the specific mechanism of the anti-tumor effect of Curcuma zedoaria and its active ingredients is still unclear, which is worthy of further research and verification.

\section{Funding}

Guangxi Natural Science Foundation Project (No. 2018GXNSFAA294115); Guangxi Natural Science Foundation Project (No. 2018GXNSFAA050064); Guangxi University of Traditional Chinese Medicine Guangxi First-Class Discipline Construction Open Project (No. 2019XK038)

\section{Disclosure statement}

The author declares no conflict of interest.

\section{References}

[1] Chen Z, Zhang Y, 2015, The Inhibitory Effect and Mechanism of Curcuma Zedoaria Polysaccharide on Lewis Lung Cancer Mice. Chinese Journal of Gerontology, 35(7): 1925-1927.

[2] Yao S, Liu B, Zhong Z, 2006, Experimental Study on the Effect of Elemene on Liver Cancer. Journal of Qiqihar Medical College, 27(3): 257-258.

[3] Ni F, 2013, Study on the Immune Mechanism of $\beta$-Elemene Combined with DC/DRibble Vaccine Against Liver Cancer in Mice. Nanjing University of Chinese Medicine.

[4] Lin L, Shen H, Wang L, 2011, The Effect of Astragaloside IV and $\beta$-Elemene on Enhancing Immune Function of Mouse Macrophages: An In Vitro Experimental Study. Chinese Journal of Clinical Laboratory Science, 29(2): 129-131.

[5] Tian L, Cao Y, Peng C, et al., 2009, Clinical Observation of the Treatment of Advanced Non-Small Cell Lung Cancer Using Elemene Emulsion Combined with Chemotherapy. Chinese Journal of Clinical Medicine, 16(5): 725-727.

[6] Tian Y, 2012, Study on the Immunogenic Apoptosis Induced by Clostridium Difficile Toxin A in 
Mouse Colon Cancer Cell CT26. South China University of Technology.

[7] Zheng L, Zhang M, Li W, 2012, Research Progress on the Correlation Between TOLL-Like Receptors and Tumor Pathogenesis. Chinese Journal of Neuro-Oncology, 10(4): 286-290.

[8] Song X, Liu H, 2020, The Role and Mechanism of Dendritic Cells Activated by the High Mobility Group Protein 1-Target of Rapamycin Pathway in Systemic Lupus Erythematosus. Chinese Journal for Clinicians, 48(7): 787-790.

[9] Wang Y, Xu D, Zhou K, et al., 2020, Dynamic Changes of TLR4 and Intestinal Flora in the Progression of Primary Liver Cancer and Their Relationship with Prognosis. Chinese Hepatology, 25(3): 273-276.

[10] Wu D, Wang Z, 2020, Research Progress of the Triggering Receptors Expressed on Myeloid Cells Family and the Etiology of Periodontitis. Journal of Oral and Maxillofacial Surgery, 30(1): 47-52

[11] Huang B, Zhao J, Li H, et al., 2005, Toll-Like Receptors on Tumor Cells Facilitate Evasion of Immune Surveillance. Cancer Res, 65(12): 5009-5014.

[12] Sato Y, Goto Y, Narita N, et al., 2009, Cancer Cells Expressing Toll-Like Receptors and the Tumor Microenvironment. Cancer Microenviron, 2(Suppl 1): 205-214.

[13] Idoyaga J, Moreno J, Bonifaz L, 2007, Tumor Cells Prevent Mouse Dendritic Cell Maturation Induced by TLR Ligands. Cancer Immunol Immunother, 56(8): 1237-1250.

[14] Hu Z, Xia X, Yang X, et al., 2014, Anti-Tumor Immunotherapy Based on Dendritic Cells. Journal of Guizhou Medical University, 39(6): 783-787.

[15] Wang L, Liu Q, Sun Q, et al., 2008, TLR4 Signaling in Cancer Cells Promotes Chemoattraction of Immature Dendritic Cells via Autocrine CCL20. Biochem Biophys Res Commun, 366(3): 852-856.

[16] Xu D, Cui N, 2013, Progress in Biotherapy of Tumors. Chinese Journal of Surgery of Integrated Traditional and Western Medicine, 19(4): 477-479.

[17] Xu Z, Zhu S, Pan N, et al., 2018, The Effect of Curcuma zedoaria Oil Produced in Guizhou on the Secretion of Toll-Like Receptors and Related Immune Factors in Rectal Cancer SW1463 Cell Line. Chinese Journal of Experimental Traditional Medical Formulae, 24(5): 137-141.

[18] Li H, Sun H, Li Y, et al., 2014, The Expression of TLR2 and TLR4 in Hepatocellular Carcinoma and Their Relationship with Proliferative Effect Factors. China Journal of Modern Medicine, 24(27): $1-8$.

[19] Street SE, Cretney E, Smyth MJ, 2001, Perform and Interferon-Gamma Activities Independently Control Tumor Initiation, Growth, and Metastasis. Blood, 97(1): 192-197.

[20] Yao S, Hu F, Liu B, 2001, The Effect of Elemene on the Immune Function of Tumor-Bearing Mice After Chemotherapy. China Pharmaceuticals, 10(8): 5.

[21] Rosenberg SA, 2014, IL-2: The First Effective Immunotherapy for Human Cancer. J Immunol, 192(12): 5451-5458.

[22] Alokail MS, Al-Daghri NM, Mohammed AK, et al., 2014, Increased TNF- $\alpha$, IL-6 and ErbB2 mRNA Expression in Peripheral Blood Leukocytes from Breast Cancer Patients. Med Oncol, 31(8): 38.

[23] Gerber SA, Sedlacek AL, Cron KR, et al., 2013, INF- $\gamma$ Mediates the Anti-Tumor Effects of Radiation Therapy in a Murine Colon Tumor. Am J Pathol, 182(6): 2345-2354.

[24] Chen Z, Xing J, Zhu J, et al., 2019, Research on the Inhibitory Effect of Curcuma zedoaria Oil on 
Transplanted Tumor of Ovarian Cancer in Nude Mice and the Synergistic Effect of its Combination with Cisplatin. Chinese Journal of Modern Applied Pharmacy, 36(12): 1462-1467.

[25] Wang J, Qi H, Lu J, et al., 2014, The Effect of Ganoderma lucidum Polysaccharides on TGF- $\beta 1$ Secreted by B16F10 Melanoma Cells. The Chinese Journal of Dermatovenereology, 28(11): 1101 1108.

[26] Xu Z, Zhu S, Luo J, et al., 2016, Effects of Curcuma zedoaria Oil on Cell Proliferation and Immune Factors of Human Rectal Cancer Cell Line SW1463. Chinese Journal of Experimental Traditional Medical Formulae, 22(13): 102-106.

[27] Moore KW, de Waal Malefyt R, Coffman RL, et al., 2001, Interleukin-10 and the Interleukin-10 Receptor. Annu Rev Immunol, 19(1): 683-765.

[28] Yin F, Zhao Y, Yao S, 2009, Expression of Raf-1 in Hepatocellular Carcinoma Tissues. World Chinese Journal of Digestology, 17(6): 618-622.

[29] Morikawa T, Matsuda H, Ninomiya K, et al., 2002, Medicinal Foodstuffs. XXIX. Potent Protective Effects of Sesquiterpenes and Curcumin from Zedoariae Rhiwma on Liver Injury Induced by Dgalactosamine/Lipopolysaccharide or Tumor Necrosis Factor-Alpha. Biol Pharm Bull, 25(5): 627631.

[30] $\mathrm{Xu} \mathrm{L}, \mathrm{Bu} \mathrm{P}$, Chen P, et al. 2007, Biological Treatment of 13 Cases of Gastric Tumors Using Curcumol-Based Tumor Vaccine. Journal of Oncology, 13(4): 303-304.

[31] Sun K, Wang L, Zhang Y, 2006, Dendritic Cell as Therapeutic Vaccines Against Tumors and its Role in Therapy for Hepatocellular Carcinoma. Cellular Molecular Immunology, 3(3): 197-203.

[32] Kang K, Zhang X, Chen Z, et al., 2015, Effect of Curcuma Zedoaria Extract on the Expression of TIMP-2, TIMP-1, nm23 and p53 in Mice with Non-Melanotic Skin Cancer. Guangdong Medical Journal, 36(17): 2666-2669. 